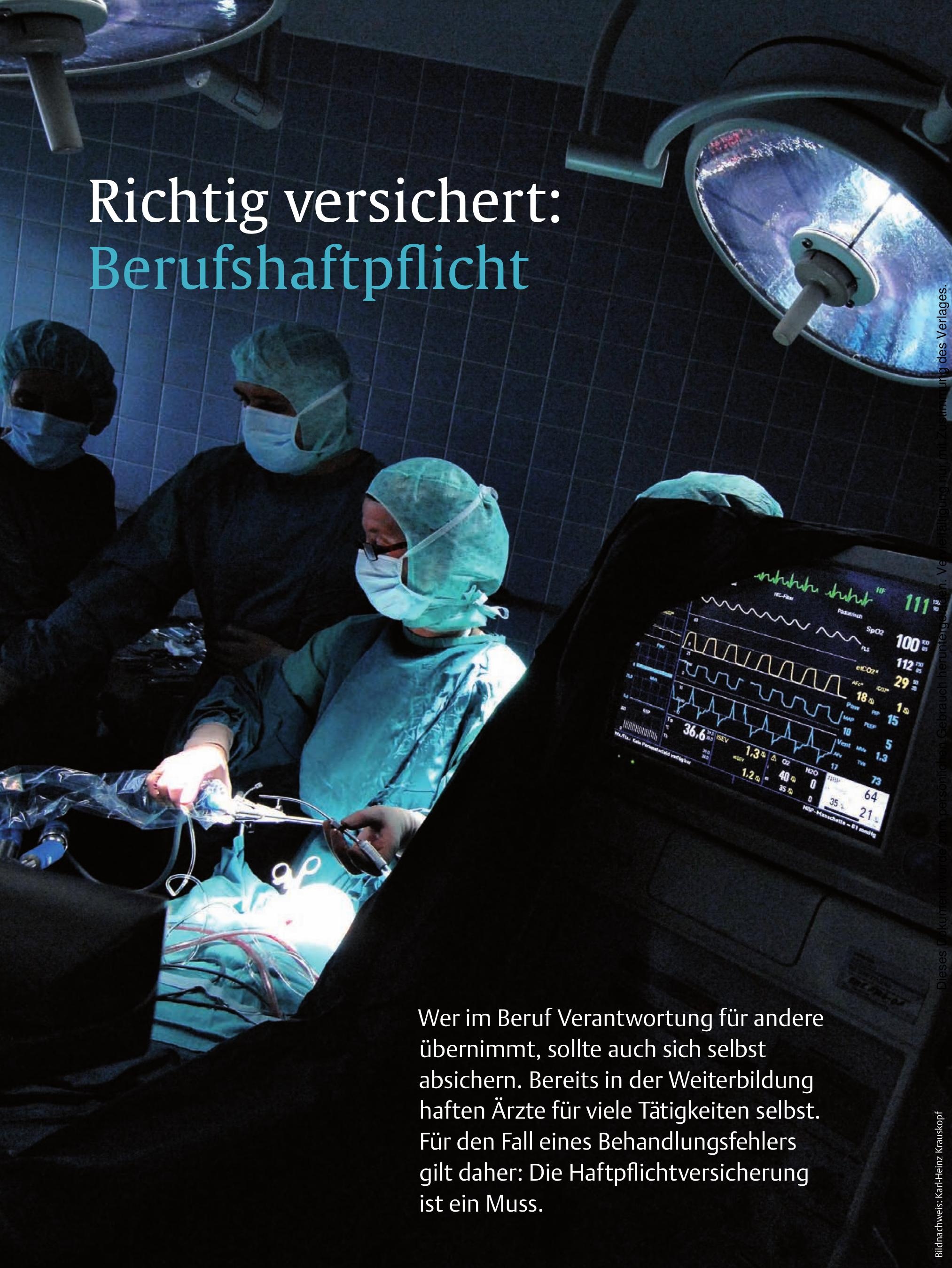


B edside-Test vergessen, Herzinfarkt nicht erkannt, Melanom übersehen: Vor solchen Fehlern graut es wohl jedem Arzt. Ärger von Patienten oder Angehörigen ist nur zu verständlich. Zumindest vor den finanziellen Folgen kann man sich aber schützen.

Versicherungspflicht Die Ärztekammern verpflichten ihre Mitglieder in §21 der Musterberufsordnung auch ausdrücklich zur Versicherung: „Ärztinnen und Ärzte sind verpflichtet, sich hinreichend gegen Haftpflichtansprüche im Rahmen ihrer beruflichen Tätigkeit zu versichern.“ Wird dem Arzt ein Behandlungsfehler vorgeworfen, prüft eine solche Versicherung die Forderungen, wehrt unberechtigte Ansprüche ab und leistet bei berechtigten Ansprüchen Schadenersatz.

\section{Haftung in der Weiterbildung}

Unter Aufsicht Für Ärzte in der Weiterbildung gibt es Versicherungspakete, die alle dienstlichen und außerdienstlichen ärztlichen Tätigkeiten sowie die private Haftpflicht umfassen. Sie werden für 55$100 €$ pro Jahr angeboten. Der Grund für dieses günstige Angebot: Wenn Ärzte in der Weiterbildung auf Anweisung oder unter Aufsicht von Ober- bzw. Chefarzt arbeiten, haften sie selten persönlich für Behandlungsfehler.

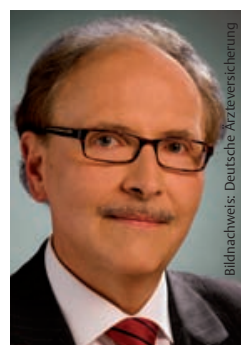

Patrick Weidinger Deutsche Ärzteversicherung „Wer Bauchtücher falsch zählt oder die Aufschriften von Blutkonserven verwechselt, haftet selbst. Auch als Weiterbildungsassistent."

Eigene Verantwortung Auf Ausnahmen weist aber Rechtsanwalt Patrick Weidinger hin, der den Bereich Arzthaftpflicht bei der Deutschen Ärzteversicherung leitet: „Für Dinge, die man seinem Ausbildungsstand nach beherrschen sollte, trägt man durchaus persönlich Verantwortung. Wer also z. B. Bauchtücher falsch zählt oder die Aufschriften von Blutkonserven verwechselt, haftet selbst.“ Ein Weiterbildungsassistent kann außerdem in die Haftung genommen werden, wenn er einen Arbeitsauftrag übernimmt, für den er noch nicht ausreichend ausgebildet ist. Hier spricht man von Übernahmeverschulden.
Praxistipp Falls Sie regelmäßig etwas tun sollen, wofür Sie noch nicht ausreichend ausgebildet sind: Suchen Sie das Gespräch mit Ihren Vorgesetzten! Neben Ihnen selbst haben auch sie die Pflicht, Übernahmeverschulden zu vermeiden.

\section{Betriebshaftpflicht}

Nicht immer vorhanden Zwar sind angestellte Ärzte für ihre Diensttätigkeit meist auch über die Betriebs-Haftpflichtversicherung ihres Krankenhausträgers versichert - doch wie so oft steckt die Tücke im Detail: Krankenhäuser sind nämlich nicht zum Abschluss einer solchen Versicherung verpflichtet, sie können das Risiko auch selbst tragen. In solchen Häusern, empfehlen Experten, sollten alle angestellten Ärzte auf jeden Fall eine private Versicherung abschließen für den Fall, dass die Klinik nicht zahlen kann [1].

Varianten der Betriebshaftpflicht Und auch wenn die Klinik versichert ist, heißt das nicht automatisch, dass der Arzt unbeschadet davonkommt. Es gibt nämlich 3 Varianten:

1. Die Versicherung schließt alle dienstlichen ärztlichen Tätigkeiten des angestellten Personals ein, nimmt aber bei grober (und anteilig bei mittlerer) Fahrlässigkeit den Arzt in Regress, d.h. verlangt den geleisteten Schadenersatz von ihm zurück.

2. Die Versicherung umfasst alle dienstlichen ärztlichen Tätigkeiten des angestellten Personals, ohne Regressanspruch.

3. Die Versicherung erstreckt sich auf alle dienstlichen und sogar außerdienstlichen ärztlichen Tätigkeiten.

Das Risiko für den Arzt, selbst haftbar gemacht zu werden, hängt also u.a. davon ab, welche Art von Betriebs-Haftpflichtversicherung sein Krankenhaus abgeschlossen hat. Kadir Yildirim, Wirtschaftsjurist beim Wirtschaftsberatungs- und Versicherungsvermittlungsdienst (WVD) des Marburger Bundes Baden-Württemberg, rät Assistenzärzten daher:

„Sehen Sie vor Abschluss einer Versicherung zunächst im Arbeitsvertrag nach und fragen Sie in der Verwaltung, was die Versicherung des Krankenhauses abdeckt. Lassen Sie sich dies schriftlich bestätigen.“
Regressforderung In einem Fall ging der Rechtsstreit um die Frage nach der zuständigen Versicherung bis vor das Bundesarbeitsgericht: Eine Weiterbildungsassistentin hatte den Bedside-Test vor einer Bluttransfusion falsch durchgeführt, die Patientin war an einer verwechselten Blutkonserve gestorben. Der Krankenhausträger zahlte zunächst an die Hinterbliebenen und an die beteiligte Krankenkasse, forderte dann aber Regress von der Ärztin wegen grober Fahrlässigkeit.

Urteil Das Bundesarbeitsgericht (Az. 8 AZR 288/96) bestätigte die Regressforderung. Begründung: „Die Beklagte hatte gleich mehrere Sicherheitsmaßnahmen missachtet, die ein Arzt bei einer Bluttransfusion zu beachten hat. [...] Die Blutübertragung gehört nicht zu den spezifischen Aufgaben eines Anästhesiearztes, sondern muss von jedem Arzt ausgeführt werden können. Schließlich war die Beklagte mit den zur Narkoseführung erforderlichen Geräten vertraut und hatte bereits bei ähnlich schwierigen Operationen als Anästhesistin gearbeitet.“ Zum Glück war die Ärztin versichert: Ihre Berufshaftpflicht übernahm die Regressforderung von 110418DM (rund 56500€).

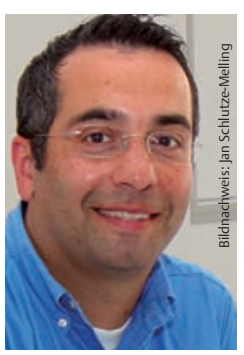

Kadir Yildirim WVD des Marburger Bundes „Die Haftpflichtversicherung hat selbst ein Interesse daran, unberechtigte Ansprüche abzuwehren. Daher übernimmt sie die Kosten von Zivilprozessen."

\section{Eigener Versicherungsbedarf}

Jenseits der Betriebshaftpflicht Klar ist: Als Weiterbildungsassistent ist man möglicherweise nicht versichert gegen Behandlungsfehler

- bei grober Fahrlässigkeit im Dienst,

- außerhalb seiner dienstlichen Tätigkeit, z.B. bei

$\triangleright$ Erste-Hilfe-Maßnahmen,

$\triangleright$ Auslandstätigkeit,

$\triangleright$ Gefälligkeitsbehandlungen von Verwandten und Freunden.

Cave Auch ein Arzt, der sich am Rande einer privaten Grillparty kurz das Kind eines Bekannten ansieht und eine Fehldiagnose trifft, haftet für diese Einschätzung! 
Wo Fehler passieren Nicht immer geht es allerdings gleich um Leben oder Tod, und nicht jeder Patient, bei dem die Behandlung nicht so gelingt wie erhofft, verklagt Arzt oder Krankenhaus. Die meisten Fehler passieren laut Statistik der Bundesärztekammer in der

- Chirurgie,

- Orthopädie und

- Inneren Medizin [2].

Spitzenreiter bei den Fehlerquellen sind Operationen, gefolgt von Fehldiagnosen [2]. Die oft zitierte Verwechslung bei Amputationen ist eine absolute Ausnahme.

Praxistipp Wenn ein Fehler passiert ist: Sprechen Sie ruhig mit dem Patienten darüber! Sie können auch Verständnis für seinen Ärger zeigen - vermeiden Sie aber Schuldeingeständnisse. Verweisen Sie dafür auf Ihre Versicherung.

\section{Schadenersatzansprüche \\ $\nabla$}

Gemeldete Vorwürfe Eine bundesweite Statistik zu Haftungsfällen fehlt bisher. Sowohl Patrick Weidinger (Deutsche Ärzteversicherung) als auch Christine Wohlers, Rechtsanwältin bei der Norddeutschen Schlichtungsstelle, gehen davon aus, dass in Deutschland jährlich etwa 40000 Behandlungsfehler gemeldet werden. In wie vielen Fällen davon sind die Vorwürfe berechtigt, d.h. liegen tatsächlich Behandlungsfehler vor? Für die Versicherung rechnet Weidinger mit 30-40\%. Bei den Fällen der Schlichtungsstelle liegt die Quote laut Wohlers bei ca. 35\%.

Beruhigend: „Ganz offensichtliche Fehler wie den übersehenen Herzinfarkt im Notdienst regulieren die Versicherer häufig schnell und ohne Streitverfahren“, so Weidinger. Auch in den übrigen Fällen einigen sich Arzt- und Patientenvertreter meist außergerichtlich, v.a. mithilfe der

\section{Tab. 1}

\section{Schadenhöhen aus Fallbeispielen der Deutschen Ärzteversicherung}

\begin{tabular}{|l|c|}
\hline Behandlungsfehler & $\begin{array}{c}\text { Schadenersatz } \\
\text { + Schmerzensgeld } \\
\text { +Verfahrenskosten }\end{array}$ \\
\hline Schwerer Geburtsschaden mit Regress der Krankenkasse & $3279000 €$ \\
\hline Schwerer Geburtsschaden & $2885000 €$ \\
\hline Nicht erkannter Herzinfarkt während eines KV-Notdienstes & $564900 €$ \\
\hline Nicht erkannter Brustkrebs & $549000 €$ \\
\hline Orthopädieschaden mit fehlender Aufklärung & $450000 €$ \\
\hline
\end{tabular}

\section{Internet} versicherung.php o www.norddeutsche-schlichtungsstelle.de
Gutachterkommissionen und Schlichtungsstellen der deutschen Ärztekammern. Dabei erlebt auch Weidinger immer wieder, dass verschiedene Gutachter eine ärztliche Maßnahme unterschiedlich beurteilen und „dass es den einzig richtigen Behandlungsstandard offenbar gar nicht gibt“.

Cave In den meisten Fällen müssen die Kläger dem Arzt einen Fehler nachweisen. Wenn sie allerdings geltend machen, vor der Behandlung nicht oder nicht ausreichend aufgeklärt worden zu sein, kann das im Zivilprozess (nicht aber im Strafprozess!) ihre Beweislast erleichtern.

Schadensummen Direkte Schadenersatzansprüche an Weiterbildungsassistenten sind zwar selten, können aber zum Ruin führen: Die oben erwähnte Regressforderung über 110418DM wurde 1988 zugesprochen. Mittlerweile haben sich die Summen teilweise vervielfacht ( Tab. 1). Am teuersten sind die seltenen, aber oft folgenreichen Behandlungsfehler in der Geburtshilfe: Bei Geburtsschäden mit bleibenden Behinderungen kann die Summe aus Schmerzensgeld, Kosten für Pflege, Lebenshaltung, Behandlungen etc. auf mehr als 3 Mio. € steigen [3].

Versicherungskonditionen für Mitglieder des Hartmannbundes:

o www.hartmannbund.de/03_mitgliederservice/versicherung.php

Finanz- und Versicherungsservice des Marburger Bundes:

o www.marburger-bund.de/marburgerbund/bundesverband/unser_service/

Berufs-Haftpflichtversicherung für Weiterbildungsassistenten bei der Deutschen Ärzteversicherung: @ www.aerzteversicherung.de/servlet/PB/menu/1116082/index.html

Schlichtungsstelle für Arzthaftpflichtfragen der Norddeutschen Ärztekammern:

\section{Versicherungsprämien} $\nabla$

Kosten Kein Wunder, dass viele Versicherer sich gar nicht auf das Geschäft mit der Arzthaftpflicht einlassen: Bei einer Internetrecherche findet man vergleichsweise wenig Anbieter. Besonders teuer ist die Versicherung für Gynäkologen, die auch Geburtshilfe leisten: „Ohne Geburtshilfe muss ein niedergelassener Gynäkologe bei der Deutschen Ärzteversicherung etwa $1800 €$ zahlen“, so Weidinger, „mit Geburtshilfe aber ein Vielfaches. Der Kunde erhält dann ein individuelles Angebot“. Während der Weiterbildung spielt die Fachrichtung dagegen keine Rolle, hier bleibt es bei den genannten 55-100€.

Auswahl Versicherer Für die individuelle Auswahl der Versicherung lohnt sich außerdem ein Blick auf die Internetseite der eigenen Ärztekammer, des Hartmannbundes, des Marburger Bundes oder der jeweiligen Fachgesellschaft: Die Verbände haben mit einigen Versicherern Gruppenverträge mit speziellen Konditionen für ihre Mitglieder ausgehandelt - auch für weitere Versicherungen wie gegen Berufsunfähigkeit. Allerdings: Wenn Sie später diesen Verband verlassen, ändern sich evtl. die Versicherungskonditionen.

Praxistipp Das Angebot für die Berufshaftpflicht beinhaltet oft auch eine private Haftpflichtversicherung - einschließlich für die eigene Familie.

\section{Wahl der Deckungssumme}

Als Deckungssumme empfehlen sowohl Yildirim als auch Weidinger

- 5 Mio.€ für Personen- und Sachschäden sowie

- 1 Mio.€ für Vermögensschäden.

Dies sind in der Regel die höchsten Versicherungssummen, die ein Versicherer anbietet. 
Praxistipp Die Kosten für die BerufsHaftpflichtversicherung sind als Werbungskosten steuerlich absetzbar.

\section{Strafrechtsschutz}

Verfahrenskosten Spätestens beim Studieren der genauen Versicherungsleistungen stolpert man über ein letztes verwirrendes Detail: den „erweiterten Strafrechtsschutz“, den die meisten BerufsHaftpflichtversicherungen einschließen. Was verbirgt sich dahinter?

Neben dem bisher dargestellten Zivilverfahren, in dem Schadenersatz und Schmerzensgeld verhandelt werden, kann es auch zu einem Strafprozess kommen: Zurückgehend auf ein Urteil von 1894, erfüllt jeder ärztliche Heileingriff den Tatbestand der Körperverletzung. Diese bleibt nur straflos, wenn der Eingriff medizinisch indiziert ist und der Patient eingewilligt hat [4]. Manche Patienten oder Angehörige erstatten daher strafrechtlich Anzeige gegen den Arzt wegen (fahrlässiger) Körperverletzung, Tötung oder unterlassener Hilfeleistung. Hier kommt der erweiterte Strafrechtsschutz zum Tragen, der als Zusatzleistung der Haftpflichtversicherung die Anwalts- und Verfahrenskosten eines Strafprozesses abdeckt.

Praxistipp Wählen Sie eine Haftpflichtversicherung mit erweitertem Strafrechtsschutz! Sonst werden nur die Kosten von Zivilprozessen übernommen, nicht aber von Strafprozessen.

Beim Zivilprozess kümmert sich die Berufs-Haftpflichtversicherung auch um entsprechende Anwälte und Gutachter, denn: „Die Versicherung hat ja ein Interesse daran, unberechtigte Ansprüche abzuwehren“, erklärt Yildirim vom WVD des Marburger Bundes.

Ziel des Strafprozesses Anders als im Zivilverfahren zielen Vorwürfe im Strafverfahren eher auf die persönliche Schuld für gesetzlich verbotene Handlungen. Der Staatsanwalt ermittelt und erhebt ggf. Anklage. Im Fall einer Verurteilung im Strafprozess bekommt der Arzt eine Haft- oder Geldstrafe, evtl. auch Berufsverbot. Wie bei anderen Straftaten muss er die Strafen selbst ableisten bzw. bezahlen. Keine Versicherung kann ihn davor schützen. Weidinger meint dazu: „Nach meiner Erfah- rung sind Strafverfahren im Verhältnis zu Zivilverfahren aber selten, und viele werden mit anwaltlicher Hilfe eingestellt.“

\section{Grenzen der „gekauften Sicherheit"}

Umfang des Schutzes Auch die beste Berufs-Haftpflichtversicherung kommt an ihre Grenzen, zum Beispiel bei

- vorsätzlicher Körperverletzung und vorsätzlicher Tötung.

Bei einigen speziellen Behandlungen sollten Sie ggf. bei Ihrer Versicherung nachfragen, ob oder inwieweit diese abgedeckt sind. Dazu zählen

- rein kosmetische, nicht medizinisch indizierte „Schönheitsoperationen“ oder

- im Heilwesen nicht anerkannte Methoden und Behandlungen wie der Offlabel-Use jenseits des geltenden Standards im Fachgebiet.

Dazu Weidinger von der Deutschen Ärzteversicherung: „Für rein kosmetische Eingriffe sind Weiterbildungsassistenten bei uns nicht versichert. Das würde sich wohl ändern, wenn solche Eingriffe in der Weiterbildungsordnung vorgesehen wären." Off-label-Use, z. B. bei austherapierten Krebspatienten oder in der Kinderanästhesie, sei bei der Deutschen Ärzteversicherung im Rahmen des versicherten Risikos abgedeckt.

Anpassung notwendig $\mathrm{Zu}$ guter Letzt wichtig zu wissen: Für den Arzt in der Weiterbildung wird eine Anpassung der Haftpflichtversicherung notwendig, sobald er

- eine Facharztprüfung ablegt,

- seine Tätigkeit ändert (z.B. kosmetische Operationen anbietet),

- freiberuflich, nebenberuflich oder als Praxisvertretung arbeitet,

- länger im Ausland arbeitet oder

- sich niederlässt.

Praxistipp Falls Sie vorübergehend beruflich aussetzen, z. B. für Kindererziehung: Führen Sie die Versicherung weiter, um Erste Hilfe oder Gefälligkeitsbehandlungen weiterhin abzudecken.

Die von den Versicherern angebotene „Nachhaftungsversicherung“ richtet sich dagegen vor allem an Ruheständler, die evtl. noch nach Ende ihrer Tätigkeit von früheren Behandlungsfehlern „eingeholt“ werden.

Julia Rojahn
Fazit Eine ausreichende Haftpflichtversicherung ist vorgeschrieben, entweder betrieblich oder privat. Mit einer eigenen Berufs-Haftpflichtversicherung können Sie sich als Weiterbildungsassistent ausreichend und relativ günstig vor Schadenersatzforderungen schützen. Daher: Versichern Sie sich - und passen Sie den Schutz Ihrem Ausbildungsstand an. 4

\section{Kernaussagen}

- Erkundigen Sie sich bei Ihrem Arbeitgeber:

$\triangleright$ Hat er eine Betriebs-Haftpflichtversicherung?

$\triangleright$ Was deckt diese genau ab?

$\triangleright$ Ist ein Regress ausgeschlossen?

$\triangleright$ Wie hoch ist die Deckungssumme?

- Prüfen Sie, ob sich eine Mitgliedschaft in einem der Ärzteverbände lohnt: Neben Beratung und Rechtsschutz für arbeitsrechtliche Prozesse bieten sie oft günstige Konditionen bei verschiedenen Versicherungen.

- Schließen Sie eine Berufs-Haftpflichtversicherung ab, die Folgendes einschließt (soweit nicht über den Krankenhausträger versichert):

$\triangleright$ dienstliche Tätigkeiten inkl. grober Fahrlässigkeit

$\triangleright$ Bereitschafts- und Notdienste außerhalb der dienstlichen Tätigkeit

$\triangleright$ Erste Hilfe

$\triangleright$ Gefälligkeitsbehandlungen von Verwandten und Freunden

$\triangleright$ Tätigkeit bei Veranstaltungen

$\triangleright$ erweiterter Strafrechtsschutz

$\triangleright$ ggf. Auslandstätigkeit

$\triangleright$ Deckungssumme: 5 Mio. $€$ für Personen- und Sachschäden, 1 Mio. $€$ für Vermögensschäden.

\section{Literatur online}

Das vollständige Literaturverzeichnis zu diesem Beitrag finden Sie im Internet:

Abonnenten und Nichtabonnenten können unter „www.thieme-connect.de/ejournals" die Seite der Lege artis aufrufen und beim jeweiligen Artikel auf „Ergänzendes Material“ klicken - hier ist die Literatur für alle frei zugänglich.

Abonnenten können alternativ über ihren persönlichen Zugang an das Literaturverzeichnis gelangen. Wie das funktioniert, lesen Sie unter: http://www.thiemeconnect.de/ejournals/help\#SoRegistrieren

Beitrag online zu finden unter http://dx. doi.org/10.1055/s-0031-1272350 